\title{
SUBSPACE BASED DOA ESTIMATION TECHNIQUES
}

\author{
Aniket R. Kale ${ }^{1}$, D. G. Ganage ${ }^{2}$, S. A. Wagh $^{3}$ \\ ${ }^{1}$ M.E Student, E\&TC Department, Sinhgad College of Engineering, Pune, Maharashtra, India \\ ${ }^{2}$ Asst. Professor, E\&TC Department, Sinhgad College of Engineering, Pune, Maharashtra, India \\ ${ }^{3}$ Asst. Professor, E\&TC Department, Sinhgad Institute of Tech. \& Science, Narhe, Pune, Maharashtra, India
}

\begin{abstract}
The subspace based techniques are used for Direction of Arrival (DOA) estimation in this work. The subspace based techniques are based on using the eigen structure of data covariance matrix. The subspace based techniques includes MUSIC, ROOTMUSIC, ESPRIT. The aim is to analyze the performance of DOA estimation algorithms in challenging environment, such as low signal to noise ratio, closely spaced sources. The performance of subspace based DOA estimation algorithm is done on Uniform Linear Array (ULA). Simulation result shows the effect of varying parameters will affect DOA estimation. The simulation shows that the MUSIC algorithm has better accuracy as compared to the Root-MUSIC and ESPRIT.
\end{abstract}

Keywords: DOA, MUSIC, Root-MUSIC, and ESPRIT

\section{INTRODUCTION}

The Direction of Arrival (DOA) estimation technique is an essential part of Smart Antenna. The antenna array which collect data from impinging signals on it with combination of spatial information by receiving signals, has the ability to estimate the angle of arrival with higher resolution algorithms.

There are four different types of DOA estimation technique conventional, maximum likelihood, subspace based and integrated techniques as given in Rappaport [7]. Beamforming techniques were used in conventional methods and it requires large number of antenna element for getting better resolution. Maximum likelihood technique requires large computation. Integrated technique is a combination of property restoral technique and subspace based technique [8]. The subspace based technique for direction of arrival (DOA) estimation, such as MUSIC, Root-MUSIC, and ESPRIT has been analyzed.

The paper is categorized as follows. Uniform linear array structure is presented in section II with mathematical formulation required for DOA estimation algorithms. Section III detailed explanation of subspace based DOA estimation algorithm. Next section IV shows the simulation result and discussion in tabular form. In section $\mathrm{V}$, some conclusion on the basis of simulation result.

\section{ARRAY SENSOR SYSTEM}

The system consists of Uniform Linear Array (ULA) as shown in Figure-1. Consider there are D signals impinges on array elements, the received input data vector at an Melements, separated by a distance $\mathrm{d}$ can be represented as a combination of the D incident signals and noise. The $u(t)$, signal vector can be defined as

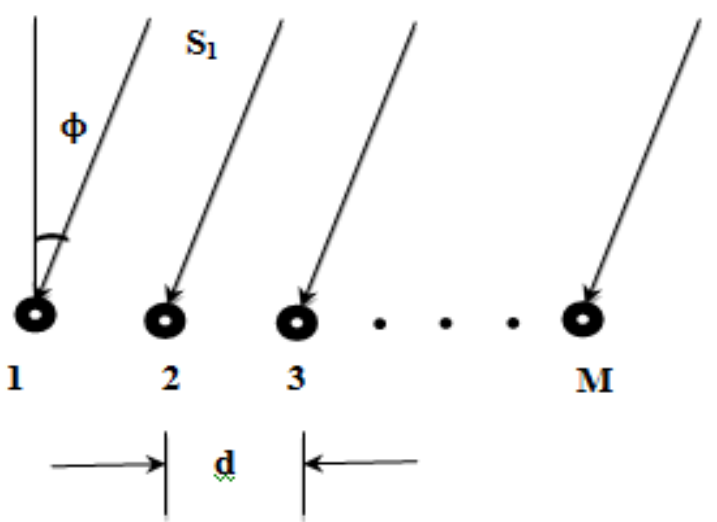

Fig-1: Uniform Linear Array

$$
\mathrm{u}(\mathrm{t})=\sum_{\mathrm{l}=0}^{\mathrm{D}-1} \mathrm{a}\left(\phi_{1}\right) \mathrm{s}_{1}(\mathrm{t})+\mathrm{n}(\mathrm{t})
$$

$$
\begin{gathered}
u(t)=\left[a\left(\phi_{0}\right) \ldots a\left(\phi_{D-1}\right)\right]\left[\begin{array}{c}
s_{1}(t) \\
\cdot \\
\cdot \\
s_{1}(t)
\end{array}\right]+n(t) \\
u(t)=A s(t)+n(t)
\end{gathered}
$$

Where, $s^{T}(t)=\left[s_{0}(t) s_{1}(t) \ldots \ldots s_{D-1}(t)\right]$ is the vector of incident signals, $n(t)=\left[n_{0}(t) n_{1}(t) \ldots \ldots n_{D-1}(t)\right]$ is the noise vector, and $\mathrm{a}\left(\phi_{\mathrm{j}}\right)$ is the array steering vector. In terms of the above data model, the input covariance matrix $R_{u u}$ can be expressed as [7]

$$
R_{u u}=A E\left[s s^{H}\right] A^{H}+E\left[n^{H}\right]
$$

Where $\mathrm{R}_{3 g}$ is the signal correlation matrix $\mathrm{E}\left[\mathrm{ss}^{\mathrm{H}}\right]$ 


\section{SUBSPACE BASED DOA ESTIMATION}

\section{ALGORITHM}

\subsection{MUSIC Algorithm}

The Multiple Signal Classification algorithm was proposed by Schmidt in 1979 .

Step 1: The array correlation matrix $\mathrm{R}_{\mathrm{uu}}$ is given as follows

$$
\mathrm{R}_{\mathrm{uu}}=\mathrm{AR}_{\mathrm{ss}} \mathrm{A}^{\mathrm{H}}+\sigma_{\mathrm{n}}{ }^{2} \mathrm{I}
$$

Next step is to find the correlational matrices eigenvalues and their associated eigenvectors. Then produce D and $\mathrm{M}-\mathrm{D}$ eigenvectors associated with the signals and noise respectively. Select the eigenvectors having smallest eigenvalues [8]. Then construct the $\mathrm{M} \times(\mathrm{M}-\mathrm{D})$ dimensional subspace composed of the noise eigenvectors such that,

$$
v_{\mathrm{n}}=\left[\mathrm{q}_{1}, \mathrm{q}_{2}, \ldots, \mathrm{q}_{\mathrm{M}-\mathrm{D}}\right]
$$

The steering vectors of array and eigenvectors of noise subspace are orthogonal to each other, $\mathrm{a}^{\mathrm{H}}(\phi) \mathrm{V}_{\mathrm{n}} \mathrm{V}_{\mathrm{n}}{ }_{\mathrm{H}}^{\mathrm{H}} \mathrm{a}(\phi)=0$ at an angle of arrival. Hence the MUSIC spectrum is given as

$$
\mathrm{P}_{\text {Music }}(\phi)=\frac{1}{\mathrm{a}^{\mathrm{H}}(\phi) \mathrm{V}_{\mathrm{n}} \mathrm{V}_{\mathrm{n}}{ }_{\mathrm{a}}^{\mathrm{H}}(\phi)}
$$

The condition of orthogonality will give rises to peaks in the spectrum of MUSIC defined in (7) by minimize the denominator.

\subsection{Root-MUSIC Algorithm}

To increase resolution performance and decrease the computational complexity various modifications have been proposed to the MUSIC algorithm. The Root-MUSIC algorithm developed by Barabell which is based on an idea of polynomial rooting giving higher resolution, but its works only if a uniform spaced linear array is present.

For the case of a uniform linear array with the spacing between each element is $d$, the $\mathbf{M}^{\text {th }}$ element of the steering vector $\mathrm{a}(\phi)$ may be expressed as

$$
a_{m}(\phi)=\exp \left(j 2 \pi\left(\frac{d}{\lambda}\right) \cos \phi\right)
$$

$\mathrm{m}=1,2, \ldots ., \mathrm{M}$.

The MUSIC spectrum given by

$$
\mathrm{R}_{\text {Music }}(\phi)=\frac{1}{\mathrm{a}^{\mathrm{H}}(\phi) \mathrm{Ca}(\phi)}
$$

$$
\begin{aligned}
\mathrm{P}_{\text {MusIC }} & -1(\phi)=\sum_{\mathbb{l}=-\mathbb{M}+1}^{\mathrm{M}+1} \mathrm{C}_{\mathrm{l}} \exp \left(-\mathrm{j} 2 \pi\left(\frac{\mathrm{dl}}{\lambda}\right) \cos \phi\right)(5) \\
D(z) & =\sum_{\mathbb{l}=-\mathbb{M}+1}^{M+1} C_{\mathbb{1}} z^{-1}
\end{aligned}
$$

Evaluating the MUSIC spectrum becomes equivalent to evaluating the polynomial $\mathrm{D}(\mathrm{z})$ on the unit circle, and the peaks in the MUSIC spectrum exist because the roots of $\mathrm{D}(\mathrm{z})$ lie close to the unit circle. Ideally, for noiseless condition, the poles will be presented exact on the unit circle at locations determined by the Direction-Of-Arrival. In other words, a pole of $\mathrm{D}(\mathrm{z})$ at $z=z_{1}=\left|z_{1}\right| \exp \left(\operatorname{jorg}\left(z_{1}\right)\right)$ will leads to a peak in the MUSIC spectrum at

$$
\cos \phi=\left(\frac{\mathrm{a}}{2 \pi d}\right) \arg \left(z_{1}\right)
$$

\subsection{ESPRIT Algorithm}

Estimation of Signal Parameters via Rotational Invariance Technique (ESPRIT) algorithm involves decomposing an $\mathrm{N}$ element array into two identical sub arrays each with $\mathrm{S}$ element. The objective of ESPRIT algorithm is to estimate the angle of arrival by determining the rotation operator.

$$
\begin{aligned}
& \mathrm{u}_{0}(\mathrm{t})=A s(t)+\mathrm{n}_{0}(\mathrm{t}) \\
& \mathrm{u}_{1}(\mathrm{t})=A \phi s(t)+\mathrm{n}_{1}(\mathrm{t})
\end{aligned}
$$

Where, $\mathrm{u}_{0}(\mathrm{t})$ and $\mathrm{u}_{1}(\mathrm{t})$ are received signal $\mathrm{n}_{0}(\mathrm{t})$ and $\mathrm{n}_{1}(\mathrm{t})$ are additive noise.

$$
\phi=\operatorname{diag}\left\{\mathrm{e}^{\mathrm{j} \psi_{1}}, \mathrm{e}^{j \Psi_{2}, \ldots,} \mathrm{e}^{j \psi_{M}}\right\}
$$

Where, $\Psi=k d \sin \theta_{\mathrm{m}} \mathrm{m}=1,2, \ldots, \mathrm{M}$

$$
\mathrm{u}(\mathrm{t})=\left[\begin{array}{l}
\mathrm{u}_{0}(\mathrm{t}) \\
\mathrm{u}_{1}(\mathrm{t})
\end{array}\right]=\overline{\mathrm{A}} \mathrm{s}(\mathrm{t})+\mathrm{n}(\mathrm{t})
$$

The input covariance matrix is given as follows:

$$
\mathrm{R}_{\mathrm{uu}}=\mathrm{AR}_{\mathrm{sg}} \mathrm{A}^{\mathrm{H}}+\sigma_{\mathrm{n}}{ }^{2} \mathrm{I}
$$

Step 2: Eigen decomposition

$$
\widehat{\mathrm{R}}_{\mathrm{uu}}=V A V^{H}
$$

Where $A=\operatorname{diag}\left\{\lambda_{0, \ldots,} \lambda_{M-1}\right\}$ and $V=\left[q_{0, \ldots,} q_{M-1}\right]$ are the eigenvalues and eigen vectors respectively.

Step 3: Estimation of the number of signals $\widehat{D}$, by using the multiplicity, $K$, of the smallest eigen value $\lambda_{\min }$, $\widehat{D}=M-K$.

Where $\mathrm{C}=\mathrm{V}_{\mathrm{n}} \mathrm{V}_{\mathrm{n}}^{\mathrm{H}}$ 
Step 4: Obtain the estimation of the signal subspace $\hat{V}_{s}=\left\lfloor\hat{V}_{0} \ldots \hat{V}_{L-1}\right\rfloor$ and decomposes it into sub-array matrix,

$$
\hat{V}_{a}=\left[\begin{array}{l}
\hat{V}_{0} \\
\hat{V}_{1}
\end{array}\right]
$$

Step 5: Compute the eigen decomposition $\left(\lambda_{0^{x} \times x} \lambda_{2 i}\right)$

$$
\hat{V}_{01}^{H} \hat{V}_{01}=\left[\begin{array}{l}
\hat{V}_{0}^{H} \\
\hat{V}_{1}^{H}
\end{array}\right]\left[\begin{array}{ll}
\hat{V}_{0}^{H} & \hat{V}_{1}^{H}
\end{array}\right]
$$

And partition $\mathrm{V}$ into $\widehat{D} \times \widehat{D}$ sub-matrices,

$$
V=\left[\begin{array}{ll}
V_{11} & V_{12} \\
V_{21} & V_{22}
\end{array}\right]
$$

Step 6: calculate the eigen values of $\Psi_{1}=-V_{12} V_{22}{ }^{-1}$

$$
\widehat{\phi}_{K}=\text { eigenvalues of }\left(-V_{12} V_{22}{ }^{-1}\right)
$$

Where, $\mathrm{K}=0, \ldots, \hat{\mathrm{L}}-1$

Step 7: Estimate the Angle-of-Arrival as

$$
\theta_{\mathrm{K}}=\cos ^{-1}\left[\frac{\arg \left(\widehat{\phi}_{\mathrm{K}}\right)}{\beta d}\right]
$$

ESPRIT gives the direction of arrival estimation in terms of the eigen values.

\section{SIMULATION RESULTS}

\subsection{MUSIC Algorithm}

The MUSIC algorithm of DOA estimation is simulated using MATLAB. In these simulations, 10 elements of ULA is consider which are equally separated by the distance of $\lambda 2$. The noise is ideal Gaussian white noise, $\mathrm{SNR}=20 \mathrm{~dB}$ and number of snapshots is 200 . The simulation has been run for two independent narrow band signals with equal amplitude, angle of arrival is -200 and 300 . The performance has been analyzed for different value of snapshots, array elements and SNR.

\section{Case.1: Effect of Varying Number of Array Elements on MUSIC Spectrum}

The three different values of the number of array elements are $\mathrm{M} 1=10, \mathrm{M} 2=50$ and $\mathrm{M} 3=100$, also other condition remain as it is. Fig-2, shows that as the value of array elements increases, the spectral beam width becomes narrower.

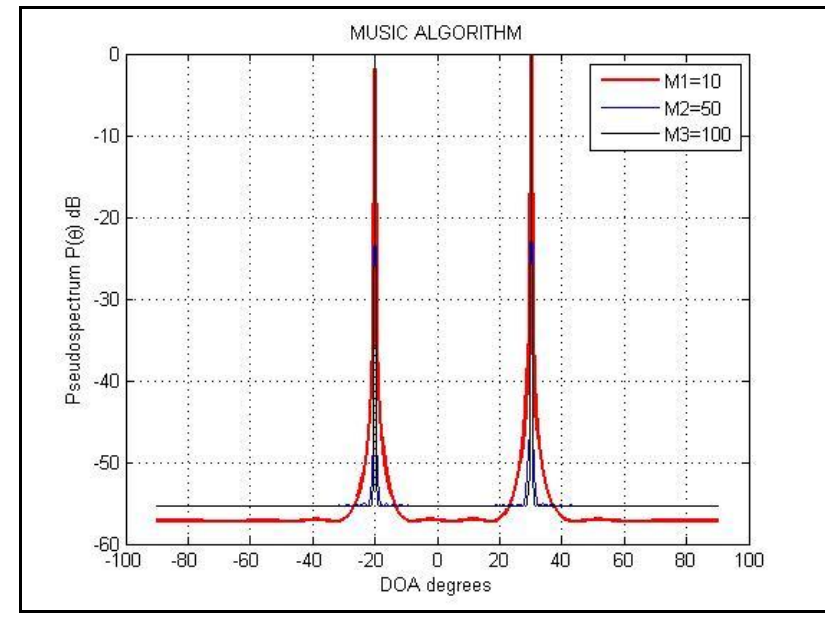

Fig-2: MUSIC spectrum for $\mathrm{M} 1=10, \mathrm{M} 2=50, \mathrm{M} 3=100$

\section{Case.2 Effect of Varying Number of Snapshots on}

\section{MUSIC Spectrum}

The three different values of the number of snapshots are $\mathrm{N} 1=20, \mathrm{~N} 2=100$ and $\mathrm{N} 3=200$ and without changing the other condition. As the number of snapshot increases the beam width of the spectrum becomes narrow while accuracy increases. Hence, the number of snapshots can be increased for accurate DOA estimation of signal.

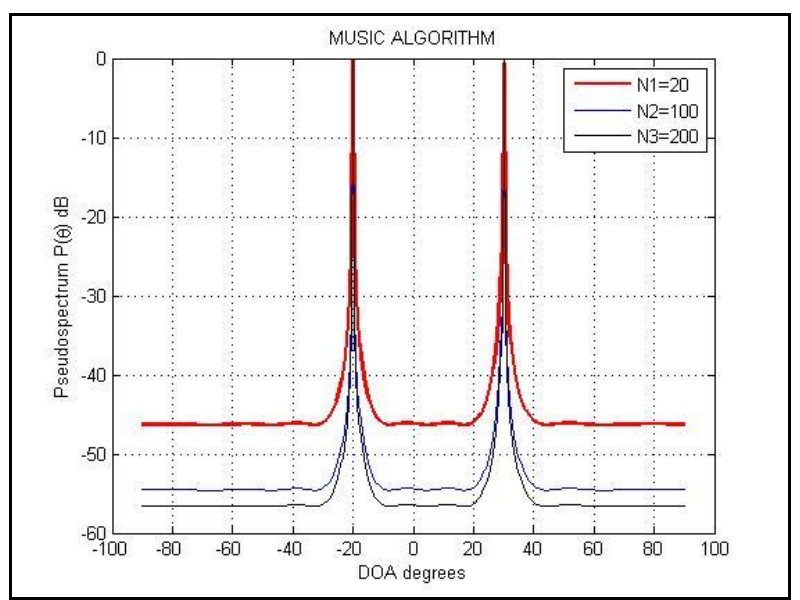

Fig-3: MUSIC spectrum for N1=20, N2=100 and N3=200

\section{Case.3: Effect of Varying Signal to Noise Ratio on MUSIC Spectrum}

The three different values of the signal to noise ratio are SNR1 $=10$, SNR2 =50 and SNR3=100, again other condition remains constant. It is clear from Fig-4 that as the value of SNR increases, the spectral beam width becomes narrower the direction of the signal becomes clearer. The accuracy of DOA estimation can be increased by increasing SNR. This implies that the performance of the MUSIC algorithm was affected by the value of SNR. 


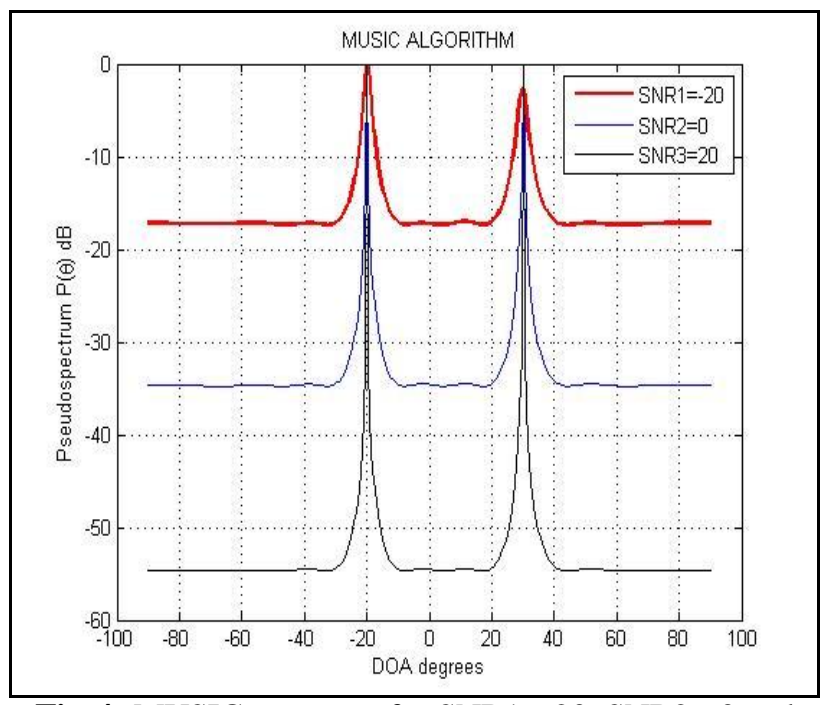

Fig-4: MUSIC spectrum for SNR1=-20, SNR2=0 and $\mathrm{SNR} 3=20$

\subsection{Root-MUSIC algorithm}

The Root-MUSIC algorithm of DOA estimation is simulated using MATLAB. In these simulations, 10 elements of ULA is consider which are equally separated by the distance of 12. The ideal Gaussian white noise was considered in this simulation. The simulation has been run for four independent narrow band signals with different angle of arrival and performance of each independent signal has been analyzed for different values of snapshots and SNR.

Table.1: Root-MUSIC for varying snapshot

\begin{tabular}{|l|l|l|l|l|}
\hline DOA & $\mathbf{0 ~ d B}$ & $\mathbf{1 0} \mathbf{~ d B}$ & $\mathbf{2 0} \mathbf{d B}$ & $\mathbf{3 0}$ dB \\
\hline $\mathbf{1 4}^{\mathbf{0}}$ & 13.8917 & 13.9186 & 13.9987 & 13.9989 \\
\hline $\mathbf{2 3}^{\mathbf{0}}$ & 23.1703 & 22.9479 & 22.9857 & 22.9998 \\
\hline $\mathbf{3 5}^{\mathbf{0}}$ & 34.8885 & 35.0784 & 34.9882 & 34.9991 \\
\hline $\mathbf{5 5}^{\mathbf{0}}$ & 55.4278 & 54.8934 & 54.9763 & 54.9983 \\
\hline
\end{tabular}

From Table 1, as the number of snapshot increases the algorithm gives accurate estimation of direction of arrival.

Table.2: Root-MUSIC for varying SNR

\begin{tabular}{|l|l|l|l|l|}
\hline DOA & $\mathbf{0 ~ d B}$ & $\mathbf{1 0} \mathbf{~ d B}$ & $\mathbf{2 0} \mathbf{d B}$ & $\mathbf{3 0}$ dB \\
\hline $\mathbf{1 4}^{\mathbf{0}}$ & 13.8917 & 13.9186 & 13.9987 & 13.9989 \\
\hline $\mathbf{2 3}^{\mathbf{0}}$ & 23.1703 & 22.9479 & 22.9857 & 22.9998 \\
\hline $\mathbf{3 5}^{\mathbf{0}}$ & 34.8885 & 35.0784 & 34.9882 & 34.9991 \\
\hline $\mathbf{5 5}^{\mathbf{0}}$ & 55.4278 & 54.8934 & 54.9763 & 54.9983 \\
\hline
\end{tabular}

\subsection{ESPRIT Algorithm}

The ESPRIT algorithm of DOA estimation is simulated using MATLAB. In these simulations, 10 elements of ULA is consider which are equally separated by the distance of 22. The ideal Gaussian white noise is considered for simulation of algorithm. The simulation has been run for four independent narrow band signals with different angle of arrival and performance of each independent signal has been analyzed for different values of snapshots and SNR.
Table.3: ESPRIT for varying snapshot

\begin{tabular}{|l|l|l|l|l|}
\hline DOA & $\mathbf{1 0}$ & $\mathbf{5 0}$ & $\mathbf{1 0 0}$ & $\mathbf{2 0 0}$ \\
\hline $\mathbf{1 4}^{\mathbf{0}}$ & 14.0265 & 13.9676 & 13.9223 & 14.0080 \\
\hline $\mathbf{2 3}^{\mathbf{0}}$ & 22.9250 & 22.9413 & 22.9494 & 23.0563 \\
\hline $\mathbf{3 5}^{\mathbf{0}}$ & 34.9665 & 35.0124 & 35.0710 & 35.0144 \\
\hline $\mathbf{5 5}^{\mathbf{0}}$ & 54.9639 & 55.1145 & 54.9493 & 54.9963 \\
\hline
\end{tabular}

Table.4 shows the effect of varying SNR on DOA estimation

Table.4: ESPRIT for varying SNR

\begin{tabular}{|l|l|l|l|l|}
\hline DOA & $\mathbf{0 ~ d B}$ & $\mathbf{1 0} \mathbf{~ d B}$ & $\mathbf{2 0} \mathbf{~ d B}$ & 30 dB \\
\hline $\mathbf{1 4}^{\mathbf{0}}$ & 13.8917 & 13.9186 & 13.9987 & 13.9989 \\
\hline $\mathbf{2 3}^{\mathbf{0}}$ & 23.1703 & 22.9479 & 22.9857 & 22.9998 \\
\hline $\mathbf{3 5}^{\mathbf{0}}$ & 34.8885 & 35.0784 & 34.9882 & 34.9991 \\
\hline $\mathbf{5 5}^{\mathbf{0}}$ & 55.4278 & 54.8934 & 54.9763 & 54.9983 \\
\hline
\end{tabular}

Hence, the number of snapshots and SNR can be increased for accurate DOA estimation of signal.

\section{CONCLUSION}

This paper includes the result of DOA estimation using MUSIC, Root-MUSIC and ESPRIT algorithms of the signal at the ULA. The algorithms were found to be sensitive to the array elements, snapshots, SNR. The simulation result also shows that the resolution of the algorithm is increasing with increasing the number of array elements, number of snapshots, SNR. The MUSIC algorithm is accurate as compared to the Root-MUSIC and ESPRIT algorithm, but has wide radiation pattern. Also, for coherent sources MUSIC algorithm is not as efficient as Root-MUSIC and ESPRIT algorithm.

\section{ACKNOWLEDGEMENTS}

The work presented in this paper has been possible due to support and availability of resources from the Sinhgad College of Engineering, Vadgaon (Bk), Pune. This work has been carried out in research and development laboratory in Department of Electronics and Telecommunication.

\section{REFERENCES}

[1] M. M. Abdalla, M. B. Abuitbel, M. Hassan, "Performance Evaluation of Direction of Arrival Estimation Using MUSIC and ESPRIT Algorithms for Mobile Communication Systems" IEEE 2013.

[2] L. Godara, "Application of Antenna Arrays to Mobile Communications, Performance Improvement, Feasibility, and System Considerations" Proceedings of the IEEE, 1997.

[3] R. O. Schmidt, "Multiple Emitter Location and Signal Parameter Estimation," IEEE, AP- 34, issue 3, pp. 276-280, Mar. 1986.

[4] R. Sanudin, N. Noordin, A. El-Rayis, N. Haridas, "Capon-Like DOA Estimation Algorithm for Direction Antenna Arrays” IEEE, 2011. 
[5] M. Mohanna, M. Rabeh, E. Zieur, S. Hekala, "Optimization of MUSIC algorithm for angle of arrivals estimation in Wireless communication" National Research Institute of Astronomy and Geophysics, ELSEVIER, 2013.

[6] C. R. Dongarsane, A. N. Jadhav, "Simulation Study on DOA estimation using Music algorithms", IJTES Vol.2, 2011.

[7] Joseph C. Liberti. Jr. Theodore S. Rappaport, "Smart Antenna for wireless communications" Prentice Hall PTR, 1999, ISBN 0-13-719287-8.

[8] Frank Gross, "Smart Antennas for Wireless Communications" McGraw-Hill, 2005.

[9] C. A. Balanis, "Antenna Theory Analysis and Design”, Wiley Pvt. Ltd, 2005.

[10] Chen Z., Gokeda G., Yu Y., "Introduction to Direction-of-Arrival Estimations" Artech House, 2010 . 